\title{
Anomalously low barrier for water dimer diffusion on $\mathrm{Cu}(111)$
}

Cord Bertram ${ }^{a, b}$, Wei Fang ${ }^{c, d, e}$, Phillipp Pedevilla ${ }^{c, d}$, Angelos Michaelides ${ }^{c, d}$, Karina Morgenstern ${ }^{a *}$

${ }^{a}$ Physical Chemistry I, Department for Chemistry and Biochemistry, Ruhr-Universität Bochum, D-44780 Bochum, Germany, ${ }^{b}$ Faculty of Physics, University of Duisburg-Essen, Lotharstr. 1, D-47057 Duisburg, Germany, ${ }^{c}$ Thomas Young Centre, London Centre for Nanotechnology, London WC1E 6BT, UK, ${ }^{d}$ Department of Physics and Astronomy, University College London, London WC1E 6BT, UK, ${ }^{e}$ Laboratory of Physical Chemistry, ETH Zurich, CH-8093 Zurich, Switzerland

E-mail: karina.morgenstern@rub.de, phone:+49-234-32-25529, fax:

+49-234-32-14182; angelos.michaelides@ucl.ac.uk;phone:+44-20-7679-0647

Keywords: diffusion | water | STM | DFT | hydrogen bonds | adsorption energy 


\begin{abstract}
A molecular-scale description of water and ice is important in fields as diverse as atmospheric chemistry, astrophysics, and biology. Despite detailed understanding of water and ice structures on a multitude of surfaces, relatively little is known about the kinetics of water motion on surfaces. Here, we report a detailed study on the diffusion of water monomers, and the formation and diffusion of water dimers through a combination of time-lapsed low-temperature scanning tunnelling microscopy experiments and first-principles electronic-structure calculations on the atomically flat $\mathrm{Cu}(111)$ surface. Based on an unprecedented long-time study of individual water monomers and dimers over days we establish rates and mechanisms of water monomer and dimer diffusion. Interestingly, we find that the monomer and the dimer diffusion barriers are similar, despite the significantly larger adsorption energy of the dimer. This is thus a violation of the rule-of-thumb that relates diffusion barriers to adsorption energies, an effect that arises because of the directional and flexible hydrogen bond within the dimer. This flexibility during diffusion should be relevant also for larger water clusters and other hydrogen bonded adsorbates. Our study stresses that a molecular-scale understanding of the initial stages of ice nanocluster formation is not possible based on static structure investigations alone.
\end{abstract}


In nature, water covers most surfaces. The interaction of water with solid surfaces is thus crucial in several scientific disciplines. In particular in areas as diverse as environmental science, ${ }^{1,2}$ solvation science, ${ }^{3}$ and astrochemistry, ${ }^{4}$ supported water-ice is of the utmost importance. For this reason, the structure and properties of water and ice has been investigated in depth on a large variety of solid surfaces, including metals, oxides, semiconductors, and carbon nanotubes. ${ }^{5-8}$ Of these, water at atomically flat surfaces, e.g. $\mathrm{Au},{ }^{9} \mathrm{Pd},{ }^{10} \mathrm{Cu},{ }^{11}$ $\mathrm{Ru},{ }^{12} \mathrm{Pt},{ }^{13,14} \mathrm{Ni},{ }^{15}$ and $\mathrm{Ag}^{16}$ has received considerable attention as well-defined model systems from which molecular-scale understanding can be obtained. Such work has primarily focused on establishing the structure of metal-supported ice overlayers and nanoclusters. In contrast, the kinetics of water motion on metal surfaces, leading eventually to the formation of such ice structures, has been investigated only scarcely on the single-molecule level. ${ }^{17,18}$ This is true despite the crucial role that water diffusion plays in the assembly of the water-ice overlayer structures that form. Indeed, recent structure investigations have suggested that the formation of fractal ice structures involves not only the mobility of water monomers, but also of small clusters. ${ }^{16,19}$

In an earlier seminal experiment, it was demonstrated that water dimer diffusion on $\operatorname{Pd}(111)$ is, at $40 \mathrm{~K}$, significantly faster than monomer diffusion. ${ }^{17}$ On this surface, a water dimer consists of one water molecule directly bound via its oxygen lone pair to the metal surface. The second molecule (the hydrogen bond acceptor) sits higher above the surface and the second molecule thus interacts only scarcely with it. For the motion of the dimer, theory proposed a novel and highly competitive diffusion mechanism at the temperature of the experiment, which includes the exchange of the two molecules in terms of binding and adsorption height. ${ }^{20}$ This diffusion process can be accelerated as compared to the diffusivity of water monomers by quantum tunneling of the hydrogens within the water molecules at low temperatures. Unfortunately, the much increased diffusivity hindered a systematic temperature-dependent investigation of the dimer motion and it remains to be explored whether this process is relevant to other surfaces or at higher temperatures. Likewise for 
dimers consisting of molecules other than water, the increased diffusivity upon dimer formation has so far only been discussed on a qualitative level, ${ }^{21,22}$ and the microscopic details of these phenomena are still in question.

In this article, we report water diffusion, dimer formation and dimer diffusion on $\mathrm{Cu}(111)$. Copper is chosen as a substrate because of its importance as a catalyst for reactions including water, ${ }^{23-26}$ e.g. in water splitting and water gas shift reactions; ${ }^{27}$ further processes for which the kinetics of water are highly important. To reveal the microscopic diffusion mechanism of the monomer and the hydrogen bonded dimer, we investigate the diffusion of $\mathrm{D}_{2} \mathrm{O}$ monomers and dimers on $\mathrm{Cu}(111)$ between 23 and $29 \mathrm{~K}$ by time-lapsed scanning tunneling microscopy (STM). Both species diffuse between on-top sites of the substrate with almost negligible difference in diffusion barrier as shown by both experiment and density functional theory (DFT) calculations. Our combined study reveals that despite very different monomer and dimer adsorption energies the diffusion barriers are equivalent because of the role played by the hydrogen bond in the dimer diffusion process. The dominant dimer diffusion mechanism is different from what was previously suggested on $\operatorname{Pd}(111)$ and does not require quantum tunneling for rapid dimer diffusion. Thus fast dimer diffusion should be valid also at higher temperatures, in particular those relevant for ice formation and the above mentioned reactions.

STM measurements were performed with a low-temperature STM under ultrahigh vacuum (UHV) conditions (base pressure $2 \cdot 10^{-10}$ mbar). ${ }^{36,37}$ The single crystal $\mathrm{Cu}(111)$ surface was cleaned by several sputter-anneal-cycles; in the initial cycles by sputtering with neon ions at $1.3 \mathrm{keV}\left(3 \cdot 10^{-5}\right.$ mbar, $\left.1-2 \mu \mathrm{A}, 45 \mathrm{~min}\right)$ and annealing at $900 \mathrm{~K}$ for 20 minutes. The last sputtering was performed at a reduced energy of $650 \mathrm{eV}\left(3 \cdot 10^{-5} \mathrm{mbar}, 0.8 \mu \mathrm{A}, 20 \mathrm{~min}\right)$ followed by a flash to $600 \mathrm{~K}$. This cleaning procedure resulted in a very low concentration of surface impurities of approximately $4 \cdot 10^{-5} \mathrm{ML} . \mathrm{D}_{2} \mathrm{O}$ is purified prior to deposition by several freeze-pump-thaw-cycles till no further improvement was observed in the mass spectra of the vapour. The overall purity determined by mass spectrometry in gas phase was 
more than $98 \%$. $\mathrm{D}_{2} \mathrm{O}$ was used in this experiment to discriminate it from the residual $\mathrm{H}_{2} \mathrm{O}$ during TPD measurements. To obtain water monomers, the water had to be deposited at a temperature, at which the molecules are not mobile on the surface. As conventional liquid helium-coolable manipulators usually do not reach this temperature regime, the water was dosed in-situ with the crystal in the STM. A total coverage of $6 \cdot 10^{-4}$ BL (bilayers) was deposited in a sequence of two deposition intervals of $22 \mathrm{~s}$ and $23 \mathrm{~s}$ at a deposition rate of $8 \cdot 10^{-4} \mathrm{BL} / \mathrm{min}$ with the sample below $15 \mathrm{~K}$ at all times.

For the diffusion experiments, the temperature of the STM was raised to between $23 \mathrm{~K}$ and $29 \mathrm{~K}$ by passing a current through Zener diodes at its base plate (for details see ${ }^{36}$ ). After stabilizing to a temperature change of less than $0.1 \mathrm{~K} / \mathrm{h}$, the same spot of the surface was imaged for extended time periods up to several days at a constant repetition rate between $60 \mathrm{~s}$ and $300 \mathrm{~s}$ to create a movie. The total number of analyzed STM images was around 3000. In analysis, consecutive images of a movie were aligned by the positions of immobile surface impurities used as reference points. After alignment, the positions of the molecules were tracked semi-automatically by fitting identical two-dimensional Gaussian profiles to the molecules in all images. The same procedure has been successfully applied before in ref. ${ }^{38}$. Here, the number of analyzed jumps per data point in the Arrhenius plot was between 10,000 at lower and 2,000 at higher temperature for the monomers and around 1,000 for the dimers in the investigated temperature range. To circumvent effects of interactions via the surface state on the diffusion process, ${ }^{39}$ only molecules with distances larger than $4 \mathrm{~nm}$ from any other particle or impurity were considered in the analysis. Details of analysis procedure and temperature calibration are given in the supporting information. ${ }^{39}$

Density functional theory (DFT) calculations were performed using the VASP code, ${ }^{40}$ with the optB86b-vdW functional, which accounts for non-local van der Waals interactions which are important for this system. ${ }^{41,42}$ A plane-wave cutoff of $600 \mathrm{eV}$ was used. The metal surface was represented using a 4 -layer-thick slab in a $3 \times 3$ unit cell with a $3 \times 3 \times 1 \mathrm{~K}$-points mesh. ${ }^{43}$ The vacuum region in the $\mathrm{Z}$ direction between the periodically repeated $\mathrm{Cu}$ slabs was 
$1.4 \mathrm{~nm}$ and dipole corrections along the $\mathrm{Z}$ axis ${ }^{44}$ were applied. The climbing image nudged elastic band method ${ }^{45}$ was used to obtain the diffusion barriers, with forces optimized to below $0.1 \mathrm{eV} / \mathrm{nm}$. The top two layers of the metal slab were free to relax in the calculations. Tests with respect to the DFT setup and sensitivity to the exchange-correlation functional are provided in the SI.

Herein, the calculated adsorption energy, $E_{\text {ads }}$, is defined by

$$
E_{\mathrm{ads}}=\left|E_{\mathrm{n}\left(\mathrm{H}_{2} \mathrm{O} / \mathrm{M}\right)}-E_{\mathrm{M}}-E_{\mathrm{n}\left(\mathrm{H}_{2} \mathrm{O}\right)}\right|
$$

in which $\mathrm{n}\left(\mathrm{H}_{2} \mathrm{O}\right)$ is the number of water molecules, $E_{\mathrm{n}\left(\mathrm{H}_{2} \mathrm{O} / \mathrm{M}\right)}$ is the total energy of the $\mathrm{n}\left(\mathrm{H}_{2} \mathrm{O}\right)$ cluster adsorbed on the metal surface system, $E_{\mathrm{M}}$ is the total energy of the relaxed bare metal slab, and $E_{\mathrm{n}\left(\mathrm{H}_{2} \mathrm{O}\right)}$ is the total energy of the relaxed $\mathrm{n}\left(\mathrm{H}_{2} \mathrm{O}\right)$ cluster in the gas phase. For the adsorption energy of the water dimer, eq. 1 implies that this is defined with respect to a gas phase water dimer.

All computed barriers reported have been corrected for zero point energy (ZPE) with deuterium masses for the hydrogen and finite size effects (details see SI). Specifically, the computed diffusion barriers correspond to the barriers obtained in the $3 \times 3$ cell plus the finite size correction. As shown in the SI, the finite size correction is the difference in the diffusion barriers between the $3 \mathrm{x} 3$ and 9x9 cells obtained on frozen surface slabs. The ZPE of the initial and transition states has been calculated using the finite displacement method within the harmonic approximation. Diffusion prefactors are calculated within the context of classical transition state theory with the prefactors expressed by

$$
\left(\Pi \omega_{\mathrm{IS}}\right) /\left(2 \pi \prod \omega_{\mathrm{TS}}\right)
$$

with positive harmonic frequency $\omega_{\text {IS/TS }}$ for initial state/transition state.

We begin by discussing the preparation and formation of adsorbed water monomers. While water forms oligomers on metal surfaces due to attractive hydrogen bonds at higher 

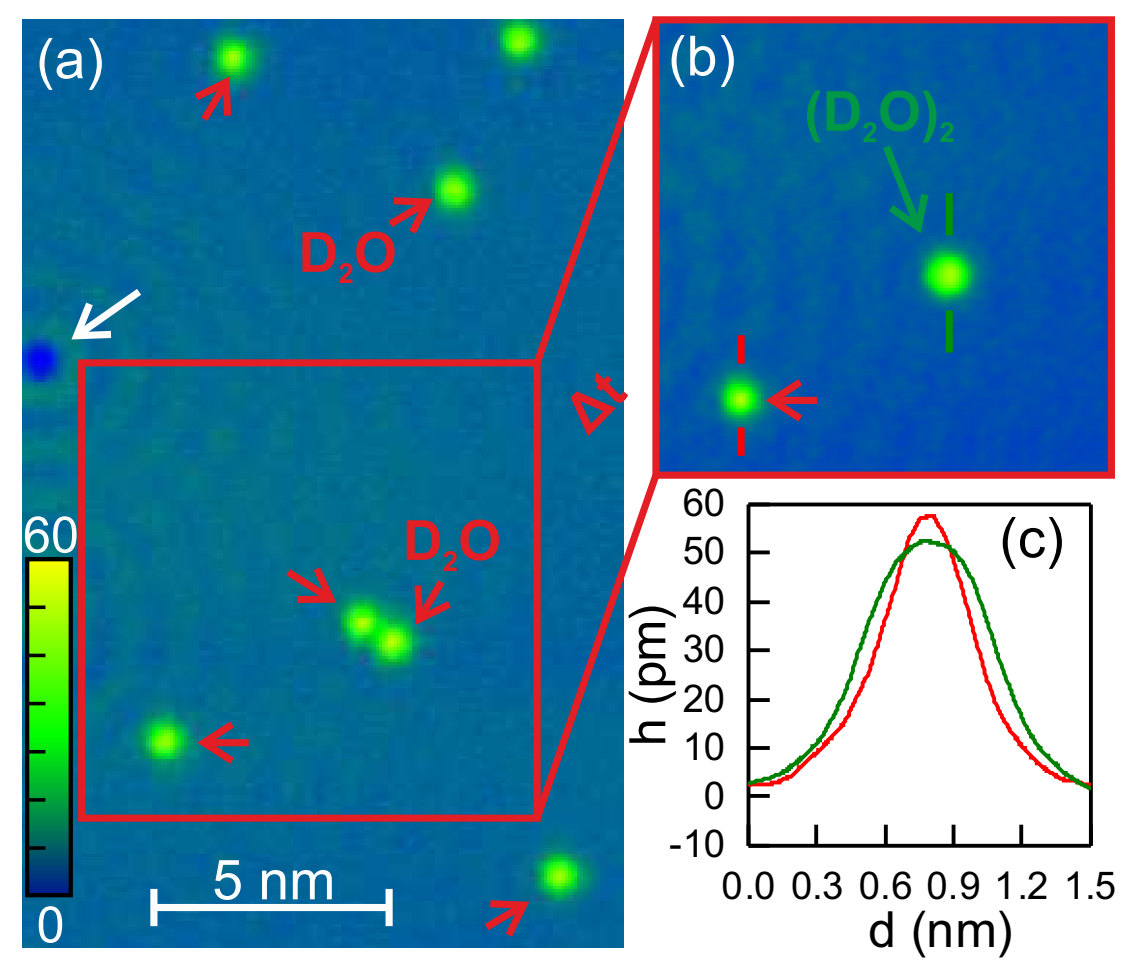

(d)
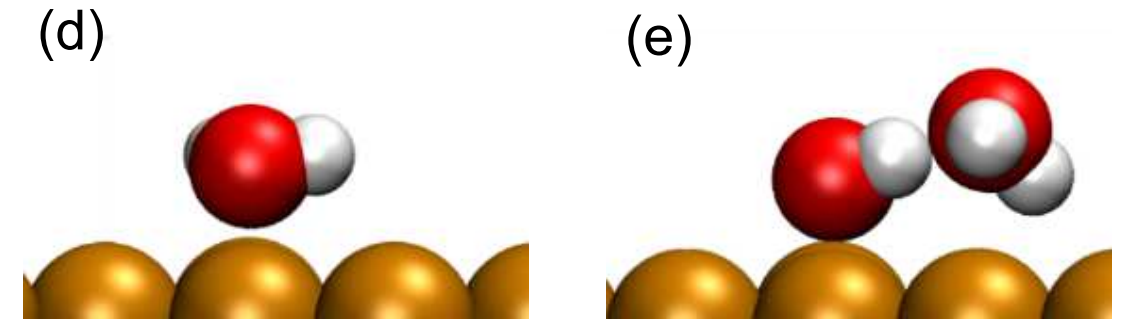

Figure 1: In-situ preparation and dimer formation: (a) Single $\mathrm{D}_{2} \mathrm{O}$ molecules after preparation (marked by red arrows), reference point marked by white arrow, z-scale in pm. (b) Subsequent image of the region in red square in (a) after $170 \mathrm{~s}$, one monomer and one dimer (marked by green arrow) $(\mathrm{V}=25 \mathrm{mV}, \mathrm{I}=20 \mathrm{pA}, \mathrm{T}=23.1 \mathrm{~K})$. (c) Apparent height profile of the monomer in red and dimer in green as marked in (b). (d,e) Side view of lowest energy adsorption energy for (d) monomer and (e) hydrogen bonded dimer; $\mathrm{Cu}$ atoms in brown, oxygen atoms in red, hydrogen atoms in white. 
adsorption temperatures,${ }^{5}$ the diffusion of the molecules is suppressed at our deposition temperature below $15 \mathrm{~K}$ leading to well separated and identical protrusions, which are randomly distributed over the surface (Fig. 1a). All protrusions exhibit a full width at half maximum $(\mathrm{FWHM})$ of $(0.81 \pm 0.02) \mathrm{nm}$ and an apparent height of $(60 \pm 3) \mathrm{pm}$. These values are within the range of values for water monomers reported previously on $\mathrm{Cu}(111)$ and on related surfaces like $\mathrm{Au}(111)$ and $\mathrm{Ag}(111)$ at similar tunneling parameters. ${ }^{28,29}$ In addition, their uniform size suggests that these single protrusions are indeed single $\mathrm{D}_{2} \mathrm{O}$ molecules. This conclusion is confirmed by the diffusion experiments below that rule out any diffusion at the deposition temperature.

Such monomers are adsorbed at on-top sites of $\mathrm{Cu}(111)$ (Fig. 1d) as calculated before ${ }^{20,28,32}$ and confirmed below by analysis of diffusion paths. Binding via the highest occupied molecular orbital (HOMO) perpendicular to the plane of the water molecule forces the molecule into an adsorption geometry with the molecular plane almost parallel to the surface (Fig. 1d).

At elevated temperatures, some of the molecules merge to create dimers, as illustrated in Fig. $1{ }^{33}$ Formation of larger oligomers is very rare at the low coverage and low temperature of our measurement. From the three monomers, marked by a square in Fig. 1a, two are located very close to each other $(<1 \mathrm{~nm}$ apart) such that the height profiles of these two molecules already overlap. In the next image, 170 seconds later, these two molecules have disappeared and a slightly broader protrusion is imaged at the spot of the molecules in the previous image (Fig. 1b). Thus, a $\mathrm{D}_{2} \mathrm{O}$ dimer has been formed and it images as a protrusion that is broader and slightly less high than the monomer. The height profile of the dimer (green in Fig. 1c) unveils an apparent height of $(49 \pm 3)$ pm and a FWHM of (1.05 \pm 0.06$)$ nm, which corresponds to an increase in area to $(170 \pm 8) \%$ as compared to the area of a monomer. In the diffusion analysis below, all protrusions with the height profile shown in Fig. 1c (green) are considered as dimers.

The round shape of the dimer is in line with DFT calculations, which revealed that the 
dimer has an asymmetric adsorption structure, with one of the water molecules (the hydrogen bond donor) adsorbed at an atop site and the other molecule (the hydrogen bond acceptor) slightly raised from the surface (Fig. 1e). This second molecule is nearly free to rotate around the surface bound molecule even at the low temperature of our measurement. ${ }^{20,30,32}$ The round shape thus reflects the time-averaged positions of this rotating species.

Note that the closest observed distance between two monomers before dimer formation is around three surface lattice distances of $\mathrm{Cu}(111)\left(\mathrm{a}_{\mathrm{Cu}(111)}=0.255 \mathrm{~nm}\right)$, i.e. $>0.7 \mathrm{~nm}$. This indicates an attractive interaction between monomers at closer distances.
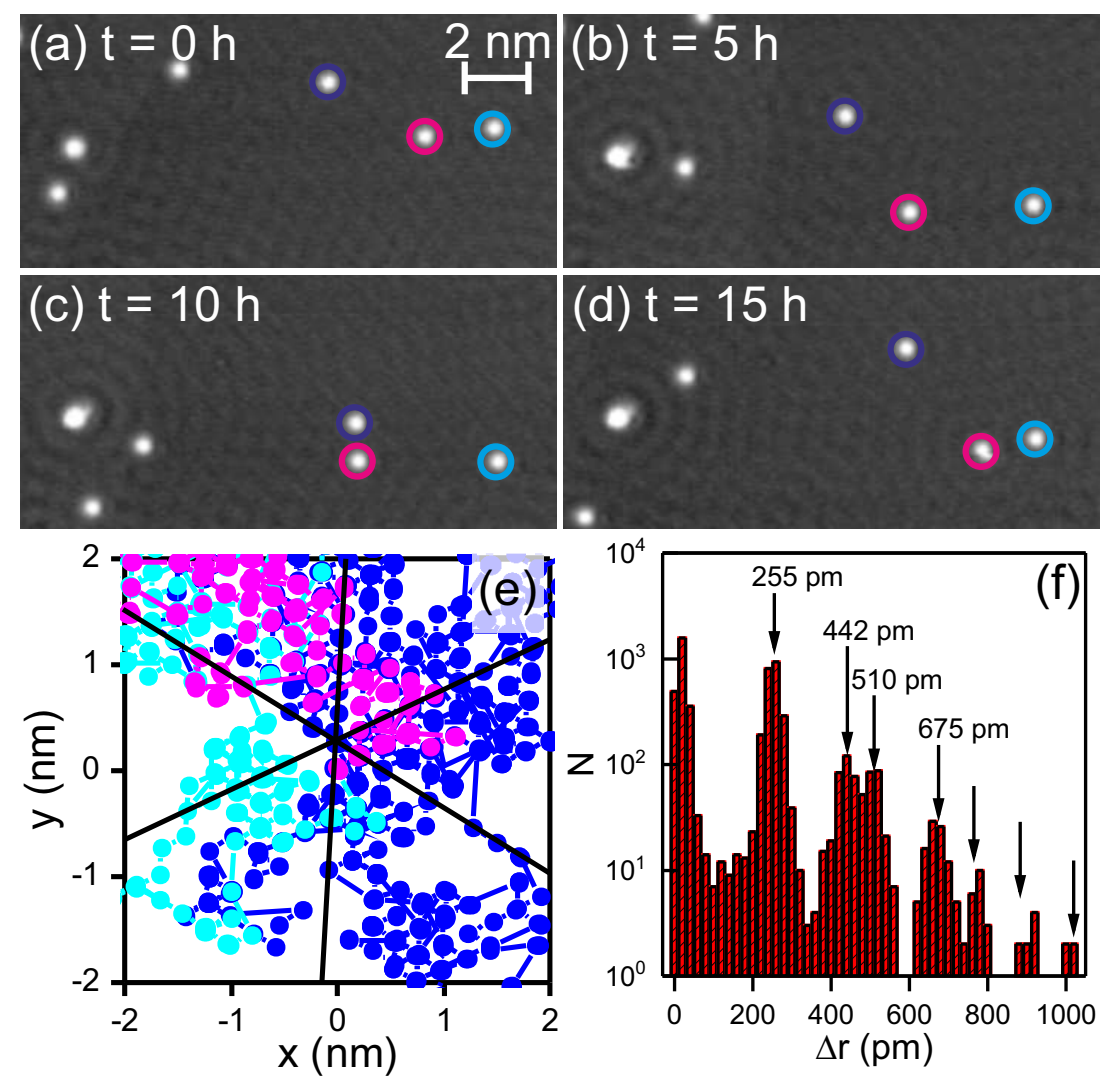

Figure 2: Diffusion of monomers: (a-d) four snapshots from a movie at (a) 0 h, (b) 5 h, (c) 10 h, (d) $15 \mathrm{~h}$ : Three molecules are encircled in different colors. (e) Cutout of tracked positions of the monomers (circles) in (a-d), black lines mark close-packed directions of $\mathrm{Cu}(111)$ as determined from images with atomic resolution. (f) Diffusion distance histogram for all positions of the movie; numbers and arrows mark characteristic atom distances on $\mathrm{Cu}(111)$; parameters: $25 \mathrm{mV}, 20 \mathrm{pA}, 26.7 \mathrm{~K}, \Delta \mathrm{t}=90 \mathrm{~s}, 853$ images in total.

Having identified monomers and dimers on the surface, we now examine how they diffuse. 
In order to make detailed measurements of diffusion rates we have identified a goldilocks temperature window of $23 \mathrm{~K}$ to $29 \mathrm{~K}$; within this temperature window the motion is fast enough that diffusion is observed on a timescale of hours to days but slow enough that cluster formation is rare. The monomer diffusion is exemplified in the tracking of molecules at $26.7 \mathrm{~K}$ in Fig. 2. The three encircled molecules in Fig. 2a to d are tracked continuously for $40 \mathrm{~h}$. A cutout of the recorded diffusion paths is displayed in Fig. 2e. The paths form a hexagonal lattice, reflecting the symmetry of $\mathrm{Cu}(111)$. This is confirmed in the diffusion distance histogram (Fig. 2f), which displays $\Delta r=\sqrt{\Delta x^{2}+\Delta y^{2}}$ with $\Delta x$ and $\Delta y$ retrieved from all positions of diffusion paths of all molecules of this movie. The FWHM of the first maximum, corresponding to no motion, serves to determine the uncertainty in the position determination. This uncertainty is, at approximately $20 \mathrm{pm}$, far below the lattice constant of $\mathrm{Cu}(111)$. The other maxima clearly correspond to the lattice distances of $\mathrm{Cu}(111)$ (indicated by arrows in Fig. 2f). A corresponding analysis of the dimer motion reveals the same symmetry and distances (see supporting information, SI). The observed diffusion maps are consistent with previous DFT calculations, which, as noted earlier, identified the ontop site as the favorable adsorption site for both monomer and dimer. ${ }^{31}$ As the diffusion lattice for monomers and dimers corresponds to $\mathrm{Cu}(111)$, the measured diffusion distances are aligned to the nearest on-top positions of $\mathrm{Cu}(111)$ for the following analysis.

Before deriving the diffusivity from the recorded data, we ensure that the motion is random: The displacements of monomers and dimers are determined from the recorded movies. We determine the mean-square displacements $\left\langle\Delta x^{2}\right\rangle$ and $\left\langle\Delta y^{2}\right\rangle$ of the diffusion for different time intervals $\Delta t$ and find the expected linear dependence. The separate diffusivities for $\Delta x^{2}$ and $\Delta y^{2}$ are identical within the error bars (see SI). Consequently, we may use the Einstein relation

$$
D=\left\langle\Delta r^{2}\right\rangle /(4 \Delta t)
$$

with $\left\langle\Delta r^{2}\right\rangle=\left\langle\Delta x^{2}\right\rangle+\left\langle\Delta y^{2}\right\rangle$ to determine the diffusivity of the molecules with $\Delta t$ the time between two subsequent images. 


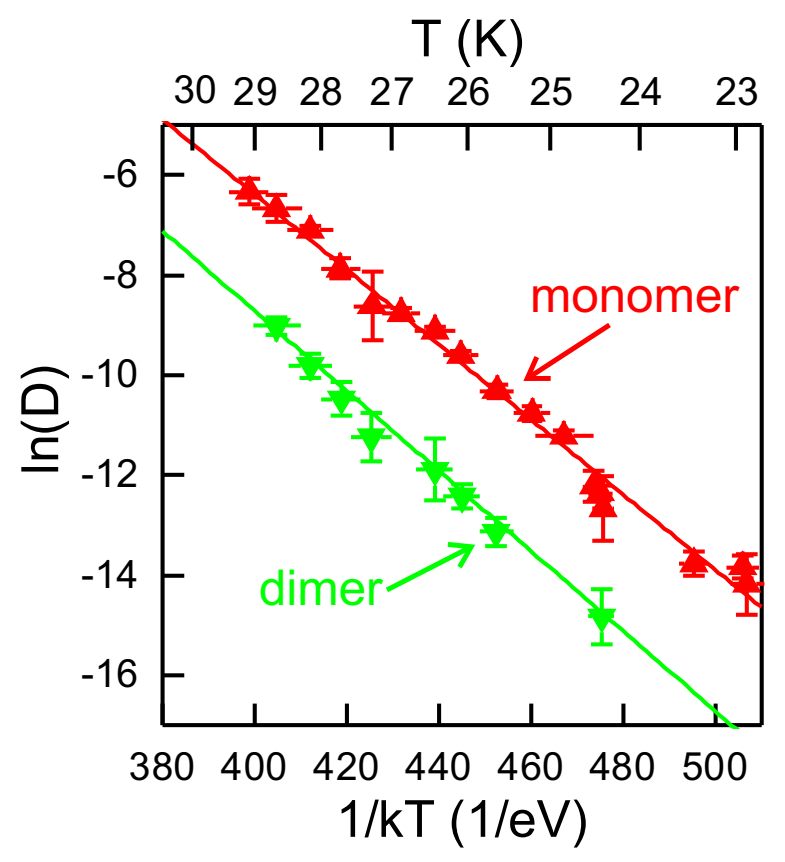

Figure 3: Arrhenius plot of experimental monomer and dimer diffusion rates versus inverse temperature: monomer in red, dimer in green, experimental data (triangles) with linear regression from resampling of experimental data (see text and SI, solid lines). Horizontal bars mark range of temperature contributing to the data point.

Our analysis reveals that at the same temperature, the diffusivity of monomers and dimers differ only by one order of magnitude. In addition, the diffusivities of both monomers and dimers follow Arrhenius behaviour within the temperature range examined, as shown in Fig. 3. Thus from the data we extract the energy barrier and diffusion prefactor using:

$$
D=D_{0, i} \cdot \exp \left(\frac{E_{i}}{\mathrm{k} T}\right)
$$

Here $E_{i}$ is the energy barrier with identifier $i$ for monomer and dimer, $T$ is the temperature, $\mathrm{k}$ is the Boltzmann constant, and the prefactor is $D_{0, i}=\alpha \cdot a_{\mathrm{Cu}(111)}^{2} \cdot \nu_{0} \cdot \exp \left(\frac{\Delta \mathrm{E}_{\mathrm{S}, i}}{\mathrm{k}}\right)$ with the dimensionality factor $\alpha=1.5$, the lattice constant $a_{\mathrm{Cu}(111)}=255 \mathrm{pm}$, the fundamental attempt frequency $\nu_{0}$, and the change in entropy $\Delta \mathrm{E}_{\mathrm{S}, i}$. For the linear regression of the data, we resample statistically the experimental data in order to correctly weight the corresponding statistical error of the data (see SI). This procedure yields an energy barrier of $E_{\text {monomer }}=$ 
$(75 \pm 4) \mathrm{meV}$ and a diffusivity of $D_{0, \text { monomer }}=1.8 \cdot 10^{10 \pm 0.7} \mathrm{~nm}^{2} / \mathrm{s}$ corresponding to a prefactor of $\nu_{0} \cdot \exp \left(\frac{\Delta E_{S, \text { monomer }}}{\mathrm{k}}\right)=1.8 \cdot 10^{11 \pm 0.7} \mathrm{~Hz}$ for the monomer. For the dimer, the corresponding values are $E_{\text {dimer }}=(80 \pm 8) \mathrm{meV}, D_{0, \text { dimer }}=1.3 \cdot 10^{10 \pm 1.4} \mathrm{~nm}^{2} / \mathrm{s}$, and $\nu_{0} \cdot \exp \left(\frac{\Delta E_{\mathrm{S}, \text { dimer }}}{\mathrm{k}}\right)=$ 5.5 $\cdot 10^{11 \pm 1.4} \mathrm{~Hz}$. Thus we find on $\mathrm{Cu}(111)$ that the diffusion barriers for the monomer and the dimer are very similar, with the difference falling within the error bars of the estimates. This is a clear violation of the rule-of-thumb that predicts a linear dependence of the diffusion energy of atoms and small molecules on several transition metal surfaces to the corresponding adsorption energy. ${ }^{35}$

(a)
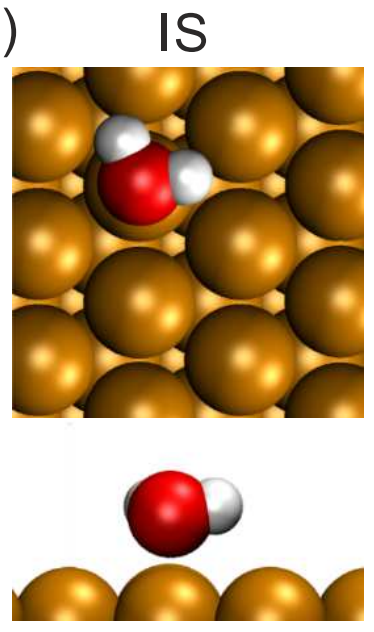

(b)
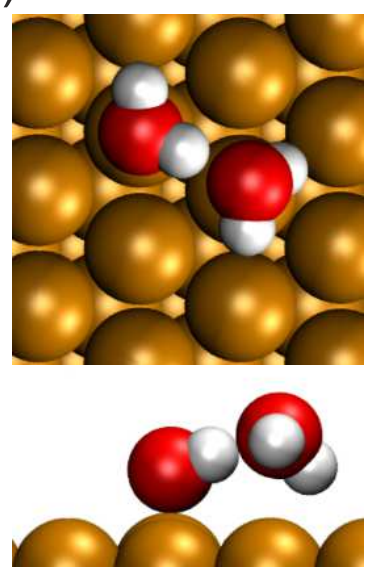

TS
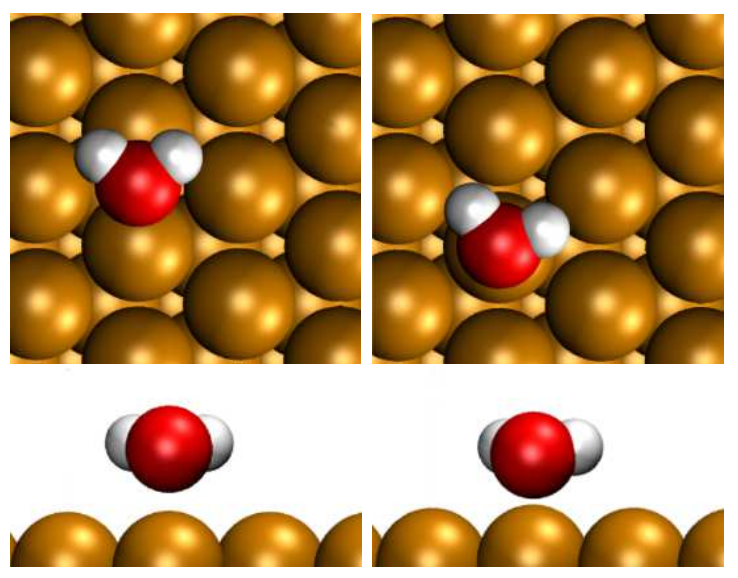

FS

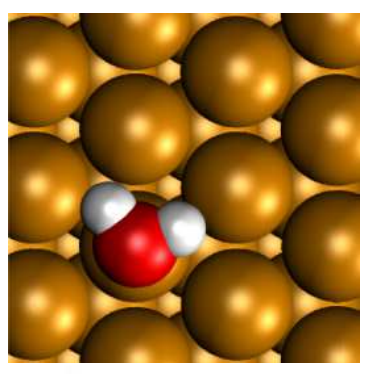

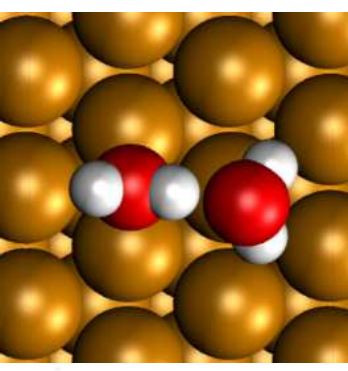
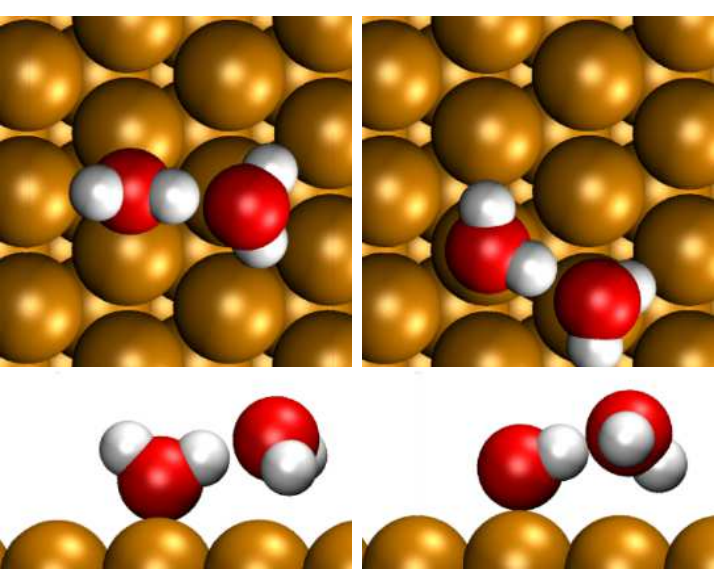

Figure 4: Lowest energy diffusion pathway for (a) monomer and (b) dimer: Top view and side view of initial (IS), transition (TS) and final state (FS); $\mathrm{Cu}$ atoms in brown, oxygen atom in red, hydrogen atom in white.

In order to understand why the diffusion barriers for monomers and dimers are so similar, 
we now turn to DFT for detailed insight into the diffusion mechanisms of each species. The most stable adsorption structures for the monomer and dimer are shown in Fig. 4a and b, respectively. The adsorption energy of the monomer is $0.35 \mathrm{eV}$ and for the dimer is $0.77 \mathrm{eV}$ (Table 1). In these adsorption structures both the monomer and the dimer are free to rotate around the surface normal with barriers $<20 \mathrm{meV}$. Various pathways for water monomer and dimer diffusion were considered with DFT, and the lowest energy pathways identified are shown in Fig. 4. We find that for both species hopping motion from on-top to on-top site via a bridge site is preferred. For the monomer, the molecule remains almost parallel to the surface throughout the entire diffusion process (Fig. 4a, TS). However, during the hopping motion of the dimer, the lower bound molecule changes to an almost upright position in the transition state (Fig. 4b, TS). The hydrogen bond to the higher bound molecule is maintained in this upright orientation, however, in the transition state, the barrier for the rotation of the higher bound molecule around the lower bound molecule is increased to $150 \mathrm{meV}$, effectively suppressing this rotation at our measurement temperature. Following the transition state the lower bound molecule returns to its almost flat lying orientation. Note that during the concerted motion of the dimer, the two molecules do not move in parallel: The lower bound molecule moves first, while the higher bound molecule simply rotates around its oxygen to maintain the hydrogen bond. Despite the more complex motion, the diffusion barrier of the dimer is, at $89 \mathrm{meV}$, only slightly larger than that of the monomer, at $86 \mathrm{meV}$ (Table $1,{ }^{34}$ ). Both of these computed barriers are in excellent agreement with experiment and consistent with the fact that the monomer and dimer diffusion barriers are the same within the error of the experimental determinations.

To understand why the dimer diffusion breaks a well established rule-of-thumb, we performed a detailed analysis of how the water-surface and water-water interactions change during the diffusion processes. Decompositions such as this are always arbitrary to some extent, however, they can be useful in providing semi-quantitative insight. ${ }^{31}$ Specifically, we considered the change in the hydrogen bond, $\mathrm{E}_{\mathrm{H}-\text { bond, }}$, the interactions between the higher 
Table 1: Comparison of theoretical (DFT) and experimental (EXP) results for water monomer and dimer adsorption and diffusion. $E_{\text {ads }}$ : Adsorption energy (as defined in the text); As noted in eqn. (1) the dimer adsorption energy is defined with respect to a gas phase water dimer, to match the definition in the adsorption-diffusion rule; $E_{d, i}$ : Diffusion barrier; $D_{0}$; Prefactor, $\nu_{0}$; Corresponding fundamental attempt frequency for $\exp \left(\frac{\Delta E_{\mathrm{S}, i}}{\mathrm{k}}\right) \approx 1$.

\begin{tabular}{|c|c|c|c|c|}
\hline & \multicolumn{2}{|c|}{ Monomer } & \multicolumn{2}{c|}{ Dimer } \\
\hline & DFT & EXP & DFT & EXP \\
\hline$E_{\text {ads }}(\mathrm{eV})$ & 0.35 & & 0.77 & \\
\hline$E_{d, i}(\mathrm{meV})$ & 86 & $75 \pm 4$ & 89 & $80 \pm 8$ \\
\hline$D_{0}\left(\mathrm{~nm}^{2} / \mathrm{s}\right)$ & $1.1 \cdot 10^{12}$ & $1.8 \cdot 10^{10 \pm 0.7}$ & $3.9 \cdot 10^{11}$ & $1.3 \cdot 10^{10 \pm 1.5}$ \\
\hline$\nu_{0}(\mathrm{~Hz})$ & $1.1 \cdot 10^{13}$ & $1.8 \cdot 10^{11 \pm 0.7}$ & $3.9 \cdot 10^{12}$ & $1.3 \cdot 10^{11 \pm 1.5}$ \\
\hline
\end{tabular}

bound molecule and the surface, $\mathrm{E}_{\text {higher-surf }}$, and between the lower bound molecule and the surface, $E_{\text {lower-surf }}$ upon going from the initial state (IS) to the transition state (TS) of the dimer diffusion process (Table 2). We find that during diffusion the interaction between the higher bound molecule and the surface barely changes ( $E_{\text {higher-surf }}$ in Table 2$)$, suggesting that despite this interaction contributing to the dimer adsorption energy, it does not contribute to the dimer diffusion barrier. Also the hydrogen bond interaction at the TS is slightly strengthened compared to the IS, an effect that serves to lower the barrier for dimer diffusion. In contrast, the interaction energy of the lower bound molecule with the surface is halved in the transition state. It is the weakening of this interaction that is the main physical origin of the dimer diffusion barrier, and it is similar to what is found for the water monomer (Table 2). Overall we see that the dimer diffusion process is intimately related to the directional but flexible hydrogen bond between the two water molecules, and it is this angular flexibility that helps to break the adsorption-diffusion energy rule-of-thumb. ${ }^{35}$

We now connect our work with earlier studies to understand why the tunneling assisted diffusion mechanism, proposed to be responsible for fast dimer diffusion on $\operatorname{Pd}(111),{ }^{17}$ does not influence the water dimer diffusion on $\mathrm{Cu}(111)$ despite the adsorption geometry being rather similar. The diffusion process on $\mathrm{Pd}(111)^{20}$ involves a facile rotation of the water dimer in the plane of the surface normal plus an exchange of the donor and acceptor molecules in the hydrogen bond. It is this hydrogen bond donor-acceptor exchange process that is facilitated 
Table 2: DFT total energy decompositions in eV of monomer and dimer diffusion barriers: Decompositions were performed on the frozen geometries of the initial (IS) and transition (TS) states of the monomer and dimer diffusion pathways and their difference (TS-IS) using the following relations: $E_{\mathrm{i} \text {-surf }}=\left|E_{i}-E_{\mathrm{H}_{2} \mathrm{O}}-E_{\text {surf }}\right|$ for $\mathrm{i}=$ monomer, higher, lower and $E_{\mathrm{H}-\text { bond }}$ $=\left|E_{\text {dimer }}-E_{\text {lower }}-E_{\text {higher }}+E_{\text {surf }}\right| . E_{i}$ is the total energy of a water molecule on $\mathrm{Cu}(111)$ fixed at the monomer, donor of the dimer (lower), or acceptor of the dimer (higher) geometry on $\mathrm{Cu}(111)$. A negative number in the last row means that the corresponding interaction contributes to increase the diffusion barrier. The TS-IS energy differences reported here are not the same as the diffusion barriers reported in Table I because zero-point energy effects and finite size corrections are not taken into account here (for details see the SI).

\begin{tabular}{|c|c|c|c|c|}
\hline & monomer & \multicolumn{3}{|c|}{ dimer } \\
\hline & $E_{\text {monomer-surf }}$ & $E_{\text {higher-surf }}$ & $E_{\mathrm{H} \text {-bond }}$ & $E_{\text {lower-surf }}$ \\
\hline IS & 0.353 & 0.168 & 0.501 & 0.312 \\
\hline TS & 0.251 & 0.165 & 0.536 & 0.155 \\
\hline TS-IS & -0.102 & -0.003 & 0.035 & -0.157 \\
\hline
\end{tabular}

by proton tunneling. Our calculations reveal that a similar donor-acceptor exchange process is possible on $\mathrm{Cu}(111)$ (see Fig. $\mathrm{S} 7$ in the supporting information). However, on $\mathrm{Cu}(111)$ the donor-acceptor exchange process has a barrier of $185 \mathrm{meV}$. This is more than twice as large as the simple dimer translation barrier of $89 \mathrm{meV}$ on $\mathrm{Cu}(111)$. On $\mathrm{Pd}(111)$, however, the dimer translation and donor-acceptor exchange barriers are rather similar, within $10 \mathrm{meV}$ of each other. We are currently examining the physical origin of the various energy barriers on Pd, $\mathrm{Cu}$, and several other metals surfaces and a detailed analysis will be published elsewhere. ${ }^{46}$ To test whether quantum tunneling influences the diffusivity of the water dimer on $\mathrm{Cu}(111)$, we calculated the crossover temperature $T_{\mathrm{c}}=\left(\hbar \omega^{\ddagger}\right) /(2 \pi \mathrm{k})$ above which the influence of tunneling on the diffusivity is negligible, with the imaginary frequency $\omega^{\ddagger}$, the Boltzmann constant $\mathrm{k}$, and the reduced Planck constant $\hbar$. From the WKB approximation point of view, assuming the barrier is parabolic-topped, $\mathrm{T}_{\mathrm{c}}$ is the temperature where tunneling starts to become favorable compared to over the barrier activation. ${ }^{47,48}$ From Feynman's path integral point of view, $T_{c}$ is the temperature at which an imaginary time path (which describes quantum statistics) starts to become delocalized around the barrier top. Hence it is an indication of when tunneling becomes important. These calculations reveal a $T_{\mathrm{c}}$ of ca. 32 $\mathrm{K}$ for the donor-acceptor exchange process. This is so close to the temperature regime 
examined experimentally that the influence of tunneling is not expected to overturn the large energetic preference for diffusion via the translation mechanism. Note that $T_{\mathrm{c}}$ for the translation mechanism is, at $12 \mathrm{~K}$, even lower. Calculations thus predict no significant influence of tunneling on the diffusion and at the temperature range of our experiments Arrhenius behavior is thus expected, which is in agreement with experiment. Furthermore, tunneling diffusion of water will clearly be negligible for any of the reactions on $\mathrm{Cu}(111)$ mentioned in the introduction at realistic reaction conditions.

Although the experiments were done with $\mathrm{D}_{2} \mathrm{O}$ dimers, it is interesting to estimate the importance of tunneling for $\mathrm{H}_{2} \mathrm{O}$ dimers. The $\mathrm{T}_{c}$ for $\mathrm{H}_{2} \mathrm{O}$ dimers is $44 \mathrm{~K}$. As expected this is higher than $\mathrm{T}_{c}$ for the $\mathrm{D}_{2} \mathrm{O}$ dimers. However, given that it remains close to the temperature regime explored in the experiments along with the large difference between the dimer translation and donor-acceptor exchange barriers, it seems unlikely that tunneling will be significant enough to alter the relative rates of $\mathrm{H}_{2} \mathrm{O}$ monomer and $\mathrm{H}_{2} \mathrm{O}$ dimer diffusion on $\mathrm{Cu}(111)$. In fact, based on a simple analysis using the WKB approximation on 1D barriers with the same barrier height and $\mathrm{T}_{c}$ as the $\mathrm{H}_{2} \mathrm{O}$ donor-acceptor-exchange process, we found that even at the lowest experimental temperature, tunneling could not make up for the large barrier difference between donor-acceptor-exchange and the translational diffusion barriers on $\mathrm{Cu}(111)$.

Our study suggests that the ice structures formed at cryogenic temperature will not largely differ, if formed in the environment on particles in the atmosphere or in astrophysics as tunneling is not important and thus just the time scale of the structure formation depends on temperature. Consequently, understanding the kinetics of structure formation enhances our understanding of the interaction of water with surfaces in several disciplines. The flexibility of the bond during diffusion will impact not only the diffusion of the dimers investigated here, but also larger clusters, even in the three-dimensional arrangement as present in solvation science, where hydrogen bonds not necessarily have to be broken during the process of solvation. We hope that our study initiates the study of hydrogen-bonded cluster to fcc(111) 
surfaces of other elements or to surfaces of different symmetry in future.

In conclusion, we have developed a molecular-scale picture of monomer and dimer diffusion of $\mathrm{D}_{2} \mathrm{O}$ on $\mathrm{Cu}(111)$. Based on a major experimental and theoretical effort diffusion barriers and mechanisms have been measured for both water monomers and dimers. The agreement between the measured and computed barriers is excellent and both experiment and simulation suggest that the monomer and dimer diffusion barriers are very similar. The fact that the monomer and dimer diffusion barriers are similar despite a substantial difference in monomer and dimer adsorption energies is an apparent contradiction of the linear adsorption-diffusion energy relationship. Analysis reveals that the breakdown of the relationship originates from the crucial role played by the directional and flexible hydrogen-bond within the dimer. In particular during the diffusion process, the interaction of the upper water with the surface and the hydrogen bond interaction barely changes. The different degrees of rotational freedom of monomer and dimer give a flexibility during their motion, which is in general expected for other hydrogen bonded oligomers. This is relevant to kinetic models of ice formation as well as clustering of hydrogen-bonded overlayer structures in general. Indeed, a multitude of rotation-diffusion combinations need to be considered in future for a proper description of the kinetics of hydrogen-bonded oligomers, or more generally for systems with bond flexibility and weak adsorbate/substrate interactions. Finally, we note that in the current system at the temperatures considered, tunneling did not play a significant role in altering the relative rates of water monomer and dimer diffusion. However, our comparison to $\mathrm{Pd}(111)$ reveals that the importance of tunneling is very much system dependent, coming down to a delicate balance of the relevant competing diffusion mechanisms. It will be interesting to understand the importance of tunneling in more detail on other substrates and for other adsorbates. We hope that the current study motivates such work in the future from both the experimental and theoretical angles. 


\section{Acknowledgments}

The research was funded by the Deutsche Forschungsgemeinschaft (DFG, German Research Foundation) under Germany's Excellence Strategy - EXC-2033 - Projektnummer 390677874. Via their membership of the UK's HEC Materials Chemistry Consortium, which is funded by EPSRC (EP/L000202), this work used the ARCHER UK National Supercomputing Service (http://www.archer.ac.uk). A. M. is supported by the European Research Council under the European Union's Seventh Framework Programme (FP/2007-2013) / ERC Grant Agreement number 616121 (HeteroIce project). We also are grateful to the UK Materials and Molecular Modelling Hub for computational resources, which is partially funded by EPSRC (EP/P020194/1), and Research-Computing at University College London (grace@UCL).

\section{Supporting information}

The supporting information provides further experimental and theoretical details. For experiment, the diffusion track and histogram of the water dimer on $\mathrm{Cu}(111)$ is given. Furthermore, the randomness of the process is proven via the Einstein relation for both, monomer and dimer motion. For theory, tests on the computational setup, the influence of the functional and finite size effects on results of the DFT calculations are explored. Moreover, the resampling of the diffusivity data for determination of the energy barrier and prefactor is explained. Then, the influence of rotation on the diffusivity is discussed, and finally the most relevant DFT structures are reported. This material is available free of charge via the Internet at http://pubs.acs.org.

Note: The authors declare no competing financial interest. 


\section{References}

(1) Liu, J.; Zhu, CQ.; Liu, K.; Jiang, Y.; Song, Y.L.; Francisco, J.S.; Zeng, X.C.; Wang, J.J. Distinct Ice Patterns on Solid Surfaces with Various Wettabilities. Proc. Nat. Ac. Sci. 2017, 114, 11285.

(2) Vergara-Temprado, J.; Miltenberger, A.K.; Furtado, K.; Grosvenor, D.P.; Shipway, B.J.; Hill A.A.; Wilkinson, J.M.; Field, P.R.; Murraya, B.J.; Carslawa, K.S. Strong Control of Southern Ocean Cloud Reflectivity by Ice-Nucleating Particles. Proc. Nat. Ac. Sci. 2018, 115, 2687.

(3) Gutberlet, A.; Schwaab, G.; Birer, Ö.; Masia, M.; Kaczmarek, A.; Forbert, H.; Havenith, M.; Marx, D. Aggregation-Induced Dissociation of $\mathrm{HCl}\left(\mathrm{H}_{2} \mathrm{O}\right)_{4}$ below $1 \mathrm{~K}$ : The Smallest Droplet of Acid, Science 2009, 324, 1545.

(4) Cleeves, L.I.; Bergin, E.A.; Alexander, C.M.O.; Du, F.J.; Graninger, D.; Oberg, K.I.; Harries, T.J. The Ancient Heritage of Water Ice in the Solar System. Science 2014, $345,1590$.

(5) Hodgson, A.; Haq, S. Water Adsorption and the Wetting of Metal Surfaces. Surf. Sci. Rep. 2009, 64, 381.

(6) Carrasco, J.; Hodgson, A.; Michaelides, A. A Molecular Perspective of Water at Metal Interfaces. Nature Mater. 2012, 11, 667.

(7) Maier, S.; Salmeron, M. How Does Water Wet a Surface? Acc. Chem. Res. 2015, 48 2783.

(8) Tomo, Y.; Askounis, A.; Ikuta, T.; Takata, Y.; Sefiane, K.; Takahashi, K. Superstable Ultrathin Water Film Confined in a Hydrophilized Carbon Nanotube. Nano Lett. 2018, 181869. 
(9) Vladimirova, M.; Stengel, M.; De Vita, A.; Baldereschi, A.; Böhringer, M.; Morgenstern, K.; Berndt, R.; Schneider, W.-D. Supramolecular Self-Assembly and Selective Step Decoration on the $\mathrm{Au}(111)$ Surface, Europhys. Lett. 2001, 56, 254.

(10) Cerda, J.; Michaelides, A.; Bocquet, M.-L.; Feibelman, P.J.; Mitsui, T.; Rose, M.; Fomin, E.; Salmeron, M. Novel Water Overlayer Growth on $\operatorname{Pd}(111)$ Characterized with Scanning Tunneling Microscopy and Density Functional Theory Phys. Rev. Lett. 2004, 93, 116101.

(11) Mehlhorn, M.; Morgenstern, K. Faceting during the Transformation of Amorphous to Crystalline Ice. Phys. Rev. Lett. 2007, 99, 246101.

(12) Gallagher, M.; Omer, A.; Darling, G.; Hodgson, A. Order and Disorder in the Wetting Layer on Ru(0001). Faraday Disc. Chem. Soc. 2009, 141, 231.

(13) Thürmer, K.; Nie, S. Formation of Hexagonal and Cubic Ice during Low-Temperature Growth. Proc. Nat. Ac. Sci. 2013, 110(29), 11757.

(14) Standop, S.; Redinger, A.; Morgenstern, M.; Michely, T.; Busse, C. Molecular Structure of the $\mathrm{H}_{2} \mathrm{O}$ Wetting Layer on $\mathrm{Pt}(111)$. Phys. Rev. B 2010, 82, 161412.

(15) Thürmer, K.; Nie, K.; Feibelman, P.J.; Bartelt, N.C. Clusters, Molecular Layers, and 3D Crystals of Water on Ni(111) J. Chem. Phys. 2014, 141, 18C520.

(16) Heidorn, S.; Bertram, C.; Morgenstern, K. The Fractal Dimension of Ice on the Nanoscale. Chem. Phys. Lett. 2016, 665, 1.

(17) Mitsui, T.; Rose, M.K.; Fomin, E.; Ogletree, D.F.; Salmeron, M. Water Diffusion and Clustering on $\operatorname{Pd}(111)$. Science 2002, 297, 1850.

(18) Heidorn, S.-C.; Bertram, C.; Cabrera-Sanfelix, P.; Morgenstern, K. Consecutive Mechanism in the Diffusion of $\mathrm{D}_{2} \mathrm{O}$ on a $\mathrm{NaCl}(100)$ Bilayer. ACS Nano 2015, 9, 3572. 
(19) Heidorn, S.; Bertram, C.; Morgenstern, K. Low-Temperature Growth of Amorphous Water Ice on $\mathrm{Ag}(111)$. J. Phys. Chem. C 2018, 122, 15304.

(20) Ranea, V.A.; Michaelides, A.; Rámirez, R.; de Andrés, P.L.; Vergés, J.A.; King, D.A. Water Dimer Diffusion on Pd(111) Assisted by an H-Bond Donor-Acceptor Tunneling Exchange. Phys. Rev. Lett. 2004, 92, 136104.

(21) Briner, B.G.; Doering, M.; Rust, H.-P.; Bradshaw, A.M. Microscopic Molecular Diffusion Enhanced by Adsorbate Interactions. Science 1997, 278, 257.

(22) Eichberger, M.; Marshall, M.; Reichelt, J.; Weber-Bargioni, A.; Auwärter, W.; Wang, R.L.C.; Kreuzer, H.J.; Pennec, Y.; Schiffrin, A.; Barth, J.V. Dimerization Boosts OneDimensional Mobility of Conformationally Adapted Porphyrins on a Hexagonal Surface Atomic Lattice. Nano Lett. 2008, 8, 4608.

(23) Gokhale, A.A.; Dumesic, J.A.; Mavrikakis, M. On the Mechanism of Low-Temperature Water Gas Shift Reaction on Copper. J. Am. Chem. Soc. 2008, 130, 1402.

(24) Andersson, K.; Ketteler, G.; Bluhm, H.; Yamamoto, S.; Ogasawara, H.; Pettersson, L.G.M.; Salmeron, M.; Nilsson, A. Autocatalytic Water Dissociation on $\mathrm{Cu}(110)$ at Near Ambient Conditions. J. Am. Chem. Soc. 2008, 130, 2793.

(25) Gawande, M.B.; Goswami, A.; Felpin, F.-X.; Asefa, T.; Huang, X.; Silva, R.; Zou, X.; Zboril, R.; Varma, R.S. Cu and Cu-Based Nanoparticles: Synthesis and Applications in Catalysis. Chem. Rev. 2016, 116, 3722.

(26) Liu, Q.; Li, J.; Tong, X.; Zhou, G. Cu and Cu-Based Nanoparticles: Synthesis and Applications in Catalysis. J. Phys. Chem. C 2017, 121, 12117.

(27) Lousada, C.M.; Johansson, A.J.; Korzhavyi, P.A. Thermodynamics of $\mathrm{H}_{2} \mathrm{O}$ Splitting and $\mathrm{H}_{2}$ Formation at the $\mathrm{Cu}(110)$-Water Interface. J. Phys. Chem. C 2015, 119, 14102. 
(28) Michaelides, A.; Morgenstern, K. Ice Nanoclusters at Hydrophobic Metal Surfaces. Nat. Mat. 2007, 6, 597.

(29) Gawronski, H.; Morgenstern, K.; Rieder, K.-H. Electronic Excitation of Ice Monomers on $\mathrm{Au}(111)$ by Scanning Tunneling Microscopy: 4Vibrational Spectra and Induced Processes. Eur. Phys. J. D 2005, 35, 349.

(30) Michaelides, A. Simulating Ice Nucleation, one Molecule at a Time, with the 'DFT Microscope'. Faraday Discuss. 2007, 136, 287.

(31) Michaelides, A.; Ranea, V.A.; de Andres, P.L., King, D.A. General Model for Water Monomer Adsorption on Close-Packed Transition and Noble Metal Surfaces. Phys. Rev. Lett. 2003, 90, 216102.

(32) Meng, S.; Wang, E.G.; Gao, S. Water Adsorption on Metal Surfaces: A General Picture from Density Functional Theory Studies. Phys. Rev. B 2004, 69, 195404.

(33) Note that on bilayer high $\mathrm{NaCl}(100)$ islands dimers do not form spontaneously, ${ }^{18}$ while on $\mathrm{Ag}(111)$ clusters form already at the deposition temperature, inhibiting a kinetics study on these surfaces.

(34) Note that the theoretical values for the diffusion barriers converge slowly with the size of the unit cell and cell sizes up to 9x9 were considered (see SI). At smaller cell sizes, periodic boundary conditions lead to a mutual interaction, reducing the diffusion energy of the monomer, but increasing the one of the dimer. This trend is consistent with experiments for the monomer, where the attraction between two water molecules becomes significant at a oxygen-oxygen distance below $1 \mathrm{~nm}\left(\approx 4 \mathrm{a}_{\mathrm{Cu}(111)}\right.$, Fig. 1a,b).

(35) Nilekar, A.U.; Greeley, J.; Mavrikakis, M. A Simple Rule of Thumb for Diffusion on Transition-Metal Surfaces. Angew. Chem. Int. Ed. 2006, 45, 7046. 
(36) Zaum, C.; Bertram, C.; Meyer auf der Heide, K.; Mehlhorn, M.; Morgenstern, K. Temperature calibration for diffusion experiments to sub-Kelvin precision. Rev. Sci. Instrum. 2016, 87, 053902.

(37) Mehlhorn, M.; Nedelmann, L.; Grujic, A.; Morgenstern, K. An Instrument to Investigate Femtochemistry on Metal Surfaces in Real-Space. Rev. Sci. Instrum. 2007, 78, 033905.

(38) Zaum, C.; Morgenstern, K. Experimental evidence for a three-body interaction between diffusing CO molecules. Nano Lett. 2016, 16, 3001.

(39) Zaum, C.; Meyer auf der Heide, K.M.; Mehlhorn, M.; McDonough, S.; Schneider, W.F.; Morgenstern, K. Differences Between Adiabatic and Non-Adiabatic Diffusion. Phys. Rev. Lett.2015, 114, 146104.

(40) Kresse, G.; Furthmüller, J. Efficient iterative Schemes for ab Initio Total-Energy Calculations Using a Plane-Wave Basis Set. Phys. Rev. B 1996, 54, 11169.

(41) Klimeš, J.; Bowler, D.R.; Michaelides, A. Van der Waals Density Functionals Applied to Solids. Phys. Rev. B 2011, 83, 195131.

(42) Klimeš, J.; Bowler, D.R.; Michaelides, A. Chemical Accuracy for the Van der Waals Density Functional. J. Phys.: Condens. Matter 2010, 22, 022201.

(43) Monkhorst, H.J.; Pack, J.D. Special Points for Brillouin-Zone Integrations. Phys. Rev. B 1976, 13, 5188 .

(44) Makov, G.; Payne, M.C. Periodic Boundary Conditions in ab Initio Calculations. Phys. Rev. B 1995, 51, 4014.

(45) Henkelman, G.; Jónsson, H. Improved tangent estimate in the nudged elastic band method for finding minimum energy paths and saddle points. J. Chem. Phys. 2000, $113,9978$. 
(46) WF́ang, P. Pedevilla, J. Richardson, X.-Z. Li, A. Michaelides, to be published.

(47) Gillan, M.J. Quantum-classical crossover of the transition rate in the damped double well. J. Phys. C 1987, 20, 3621.

(48) Mills, G.; Schenter, G.; Makarov, D.; Jonsson, H. Generalized path integral based quantum transition state theory. Chem. Phys. Lett. 1997,278, 91. 
TOC graphics:

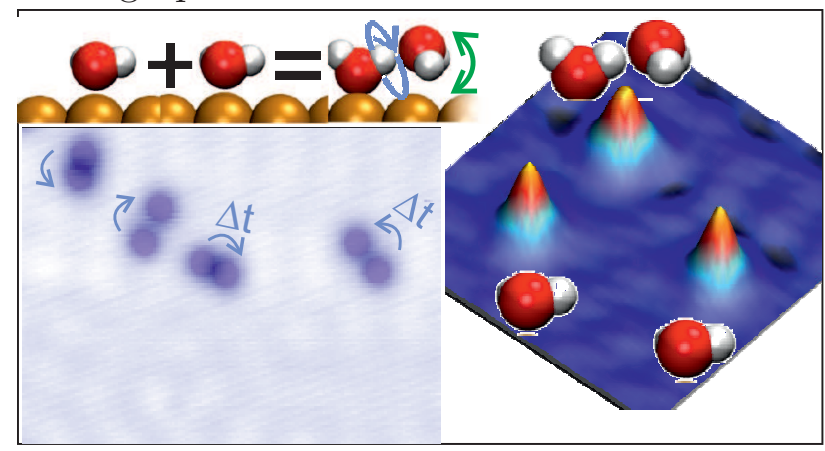

